\title{
APOLARITY FOR DETERMINANTS AND PERMANENTS OF GENERIC MATRICES
}

\author{
SEPIDEH MASOUMEH SHAFIEI
}

\begin{abstract}
We show that the apolar ideals to the determinant and permanent of a generic matrix, the Pfaffian of a generic skew symmetric matrix and the hafnian of a generic symmetric matrix are each generated in degree 2 . As a consequence, using a result of Ranestad and Schreyer, we give lower bounds to the cactus rank and rank of each of these invariants. We compare these bounds with those obtained by Landsberg and Teitler.
\end{abstract}

1. Introduction. This paper was originally motivated by a question from Zach Teitler about the generating degree of the annihilator ideal of the determinant and the permanent of a generic $n \times n$ matrix. Here annihilator is meant in the sense of the apolar pairing, i.e., Macaulay's inverse system [17]. Our main result is that the apolar ideals of the determinant and of the permanent of a generic matrix are generated in degree 2 (Theorems 2.12 and 2.13). The reason for Teitler's interest in this problem is the recent paper by Ranestad and Schreyer [19], which gives a lower bound for smoothable rank, border rank and cactus rank of a homogeneous polynomial in terms of the generating degree of the apolar ideal and the dimension of the Artinian apolar algebra defined by the apolar ideal. We apply this and our result to bounding the scheme/cactus and Waring lengths of the determinant and the permanent of the generic matrix (Theorem 3.6). In Section 4 we give the analogous result for the annihilator ideal of the Pfaffian of a generic skew symmetric matrix (Theorem 4.12 and Corollary 4.13) and the annihilator of the hafnian of a generic symmetric matrix (Theorem 4.15)

2010 AMS Mathematics subject classification. Primary 13H10, 14 N15.

Keywords and phrases. Determinant, permanent, Pfaffian, hafnian, apolar ideal, Waring rank, cactus rank, Gröbner basis.

I gratefully acknowledge support in summer 2012 from the Ling-Ma fellowship.

Received by the editors on May 14, 2013, and in revised form on January 16, 2014. 
In a sequel paper [20] we study the apolar ideal of the determinant and permanent of the generic symmetric matrix. We show an analogous result there for the determinant, but the permanent of a generic symmetric matrix is generated in degrees 2 and 3 .

Let $\mathrm{k}$ be a field of characteristic zero or characteristic $p>2$, and let $A=\left(a_{i j}\right)$ be a square matrix of size $n$ with $n^{2}$ distinct variables. The determinant and permanent of $A$ are homogeneous polynomials of degree $n$. Let $R=\mathrm{k}\left[a_{i j}\right]$ be the polynomial ring, $S=\mathrm{k}\left[d_{i j}\right]$ the ring of inverse polynomials associated to $R$, and let $R_{k}$ and $S_{k}$ denote the degree- $k$ homogeneous summands. Then $S$ acts on $R$ by contraction:

$$
\left(d_{i j}\right)^{k} \circ\left(a_{u v}\right)^{\ell}= \begin{cases}a_{u v}^{\ell-k} & \text { if }(i, j)=(u, v), \\ 0 & \text { otherwise. }\end{cases}
$$

If $h \in S_{k}$ and $F \in R_{n}$, then we have $h \circ F \in R_{n-k}$. This action extends multilinearly to an action of $S$ on $R$. When the characteristic of the field $\mathrm{k}$ is zero or char $\mathrm{k}=p$ greater than the degree of $F$, the contraction action can be replaced by the action of partial differential operators with constant coefficients ([9], [13] and Appendix A).

Definition 1.1. To each degree- $j$ homogeneous element, $F \in R_{j}$, we associate $I=\operatorname{Ann}(F)$ in $S=\mathrm{k}\left[d_{i j}\right]$ consisting of polynomials $\Phi$ such that $\Phi \circ F=0$. We call $I=\operatorname{Ann}(F)$, the apolar ideal of $F$ and the quotient algebra $S / \operatorname{Ann}(F)$ the apolar algebra of $F$.

Let $F \in R$. Then $\operatorname{Ann}(F) \subset S$ is an ideal, and we have

$$
(\operatorname{Ann}(F))_{k}=\left\{h \in S_{k} \mid h \circ F=0\right\} .
$$

Let $V$ be a vector subspace of $R$,

$$
\operatorname{Ann}(V)=\{h \in S \mid h \circ F=0 \text { for all } F \in V\} .
$$

Remark 1.2. Let $\phi:\left(S_{i}, R_{i}\right) \rightarrow \mathrm{k}$ be the pairing $\phi(g, f)=g \circ f$, and let $V$ be a vector subspace of $R_{k}$. Then we have

$$
\operatorname{dim}_{\mathrm{k}}\left(V^{\perp}\right)=\operatorname{dim}_{\mathrm{k}} S_{k}-\operatorname{dim}_{\mathrm{k}} V .
$$

For $V \subset R_{k}$, we denote by $V^{\perp}=\operatorname{Ann}(V) \cap S_{k}$.

Let $F$ be a form of degree $j$ in $R$. We denote by $\langle F\rangle_{j-k}$ the vector space $S_{k} \circ F \subset R_{j-k}([\mathbf{1 3}])$. 
We denote by $M_{k}(A)$ the vector subspace of $R$ spanned by the $k \times k$ minors of $A$.

\section{Lemma 1.3.}

$$
S_{k} \circ(\operatorname{det}(A))=M_{n-k}(A) \subset R_{n-k}
$$

Proof. It is easy to see that

$$
S_{k} \circ(\operatorname{det}(A)) \subset M_{n-k}(A) \subset R_{n-k} .
$$

For the other inclusion, let $M_{\hat{I}, \hat{J}}(A), I=\left(i_{1}, \ldots, i_{k}\right), J=\left(j_{1}, \ldots j_{k}\right)$, $1 \leq i_{1} \leq i_{2} \leq \cdots \leq i_{k} \leq n, 1 \leq j_{1} \leq j_{2} \leq \cdots \leq j_{k} \leq n$ be the $(n-k) \times(n-k)$ minor of $A$ one obtains by deleting the $I$ rows and $J$ columns of $A$. Now it is easy to see that

$$
M_{\hat{I}, \hat{J}}= \pm\left(d_{i_{1}, j_{1}} \cdot d_{i_{2}, j_{2}} \cdots d_{i_{k}, j_{k}}\right) \circ \operatorname{det}(A) .
$$

Hence, $M_{\hat{I}, \hat{J}} \in S_{k} \circ(\operatorname{det}(A))$.

Remark 1.4. (see [13, page 69, Lemma 2.15]). Let $F \in R$ and $\operatorname{deg} F=j$ and $k \leq j$. Then we have

$$
(\operatorname{Ann}(F))_{k}=\left\{h \in S_{k} \mid h \circ\left(S^{j-k} \circ F\right)=0\right\}=\left(\operatorname{Ann}\left(S^{j-k} \circ F\right)\right)_{k} .
$$

Remark 1.5. By Lemma 1.3 and Remark 1.4, we have

$$
\operatorname{Ann}(\operatorname{det}(A))_{k}=M_{k}(A)^{\perp} \text {. }
$$

Example 1.6. Let $n=3$,

$$
A=\left(\begin{array}{lll}
a_{11} & a_{12} & a_{13} \\
a_{21} & a_{22} & a_{23} \\
a_{31} & a_{32} & a_{33}
\end{array}\right), \quad D=\left(\begin{array}{lll}
d_{11} & d_{12} & d_{13} \\
d_{21} & d_{22} & d_{23} \\
d_{31} & d_{32} & d_{33}
\end{array}\right)
$$

Let $M_{i j}$ be the minor corresponding to the entry $a_{i j}$ of matrix $A$, and let $P_{i j}$ be the permanent corresponding to the entry $d_{i j}$ of matrix $D$. Note that $P_{11}=d_{22} d_{33}+d_{32} d_{23}$ annihilates $\operatorname{det}(A)=a_{11} M_{11}-a_{12} M_{12}+$ $a_{13} M_{13}$, since evidently,

$$
\begin{aligned}
P_{11} \circ a_{11} M_{11} & =\left(d_{22} d_{33}+d_{23} d_{32}\right) \circ\left(a_{11} a_{22} a_{33}-a_{11} a_{23} a_{32}\right) \\
& =a_{11}-a_{11}=0 . \\
P_{11} \circ a_{12} M_{12} & =\left(d_{22} d_{33}+d_{23} d_{32}\right) \circ\left(a_{12} a_{21} a_{33}-a_{12} a_{23} a_{31}\right)=0 .
\end{aligned}
$$




$$
P_{11} \circ a_{13} M_{13}=\left(d_{22} d_{33}+d_{23} d_{32}\right) \circ\left(a_{13} a_{21} a_{32}-a_{13} a_{22} a_{31}\right)=0 .
$$

Thus, it is easy to see that, when $n=3, P_{i j} \circ M_{k l}=0$ for each $1 \leq$ $i, j, k, l \leq 3$. So, in the case $n=3$, the annihilator of the determinant of a generic matrix certainly contains all its $2 \times 2$ permanents. It also contains all the unacceptable monomials that do not appear in any term of the determinant. See Definition 2.5. Later we show that the set of $2 \times 2$ permanents and all the degree two unacceptable monomials generate the annihilator ideal of the determinant of an $n \times n$ matrix.

\subsection{Summary of the main results.}

- We show that the following apolar ideals are generated in degree two and we specify the generators:

- Determinant of a generic $n \times n$ matrix (Theorem 2.12). This ideal is generated by $2 \times 2$ permanents and certain unacceptable degree two monomials.

- Permanent of a generic $n \times n$ matrix (Theorem 2.13). This ideal is generated by $2 \times 2$ minors and unacceptable degree two monomials.

- Pfaffian of a generic skew symmetric $2 n \times 2 n$ matrix (Theorem 4.12). This ideal is generated by certain degree two binomials corresponding to each $4 \times 4$ Pfaffian and some monomials.

- Hafnian of a generic symmetric $2 n \times 2 n$ matrix (Theorem 4.15). This ideal is generated by certain degree two binomials corresponding to $4 \times 4$ hafnians and some monomials.

The proof in each case is done by identifying the degree 2 homogeneous elements of the annihilator ideal $V(F)=$ $(\operatorname{Ann}(F))_{2}$, then showing that $(\operatorname{Ann}(F))_{n}=(V(F))_{n}$. This is Proposition 2.10 in the case where $F$ is the determinant of a generic matrix and is the key step. The study of the dual modules $S_{k} \circ F$ shows that $S_{k} \circ F$ is the span of all determinants (permanents or Pfaffians) of suitable submatrices of $A$ or $X^{s k}$. This is Lemma 1.3 for $F=\operatorname{det}(A)$ and Lemma 4.8 in the case of the Pfaffian $F$ of a generic skew symmetric $2 n \times 2 n$ matrix $X^{s k}$. Then, by applying the key step for degree $n$ to each minor, after restricting the variables, we show that $(\operatorname{Ann}(F))_{k}=(V(F))_{k}$ 
for each $3 \leq k \leq n$ (Corollaries 2.11 and 4.11, respectively, for the determinant of a generic matrix $A$ and the Pfaffian of $X^{s k}$ ).

- We apply these results to give a lower bound for:

- Cactus rank of the determinant and permanent of the generic $n \times n$ matrix (Theorem 3.6).

- Cactus rank of the Pfaffian of a generic skew symmetric matrix (Corollary 4.13) and of the hafnian of the generic symmetric matrix (Theorem 4.15).

- We give a Gröbner basis of homogeneous quadrics for the apolar ideal of the determinant (permanent) of a generic matrix consisting of the $2 \times 2$ permanents (determinants) and the degree two unacceptable monomials (Theorem 5.3). In particular, the algebras $S /(\operatorname{Ann}(\operatorname{det}(A))$ and $S /(\operatorname{Ann}(\operatorname{perm}(A))$ are Koszul (see [8, page 2]).

2. The apolar algebras associated to the $n \times n$ generic matrix. In this section, we determine the annihilator ideals of the determinant and the permanent of a generic $n \times n$ matrix. In subsection 2.1, we review the dimension of the subspace of $k \times k$ minors and permanents of an $n \times n$ generic matrix. In subsection 2.2, we determine the generators of the apolar ideal to the determinant and permanent of a generic matrix.

We continue to employ the notation of Section 1 , so $R=\mathrm{k}\left[a_{i j}\right]$ is a polynomial ring, $S=\mathrm{k}\left[d_{i j}\right]$ is the ring of inverse polynomials associated to $R$ and $S$ acts on $R$ by contraction.

2.1. Hilbert function and dimension of spaces of minors and permanents. Denote by $\mathfrak{A}_{A}=S /(\operatorname{Ann}(\operatorname{det}(A))$ the apolar algebra of the determinant of the matrix $A$. Recall that the Hilbert function of $\mathfrak{A}_{A}$ is defined by $H\left(\mathfrak{A}_{A}\right)_{i}=\operatorname{dim}_{\mathbf{k}}\left(\mathfrak{A}_{A}\right)_{i}$ for all $i=0,1, \ldots$.

Definition 2.1. Letting $F$ be a polynomial in $R$, we define $\operatorname{deg}(\operatorname{Ann}(F))$ to be the length of $S / \operatorname{Ann}(F)$.

The number of the $k \times k$ minors and permanents of a generic $n \times n$ matrix is $\left(\begin{array}{l}n \\ k\end{array}\right)^{2}$. The $k \times k$ minors form a linearly independent set ([4, Theorem 5.3, Remark 5.4]), and the $k \times k$ permanents form another linearly independent set. To show the linear independence of these 
two sets we choose a term order, for example the diagonal order (see Definition 5.1) where the main diagonal term is a Gröbner initial term. Now the initial terms give a basis for the two spaces ([16, page 197]). So the dimension of the space of $k \times k$ minors of an $n \times n$ matrix and the dimension of the space of $k \times k$ permanents of an $n \times n$ matrix are both $\left(\begin{array}{l}n \\ k\end{array}\right)^{2}$. By Lemma 1.3 and Remark 1.5, we have

$$
H(S / \operatorname{Ann}(\operatorname{det} A))_{k}=H(S / \operatorname{Ann}(\operatorname{Perm} A))_{k}=\left(\begin{array}{l}
n \\
k
\end{array}\right)^{2} .
$$

So the length $\operatorname{dim}_{\mathbf{k}}\left(\mathfrak{A}_{A}\right)$ satisfies

$$
\operatorname{dim}_{\mathrm{k}}\left(\mathfrak{A}_{A}\right)=\sum_{k=0}^{k=n}\left(\begin{array}{l}
n \\
k
\end{array}\right)^{2}=\left(\begin{array}{c}
2 n \\
n
\end{array}\right) .
$$

A combinatorial proof of equation (2.2) can be found in [21, Example 1.1.17].

2.2. Generators of the apolar ideal. In this section, we determine the generators of the apolar ideal of the determinant and permanent of a generic matrix.

Notation. For a generic $n \times n$ matrix $A=\left(a_{i j}\right)$, the permanent of $A$ is a polynomial of degree $n$ defined as follows:

$$
\operatorname{Per}(A)=\sum_{\sigma \in S_{n}} \Pi a_{i, \sigma(i)} .
$$

Lemma 2.2. Let $A=\left(a_{i j}\right)$ be a generic $n \times n$ matrix. Then each $2 \times 2$ permanent of $D=\left(d_{i j}\right)$ annihilates the determinant of $A$.

Proof. Assume we have an arbitrary $2 \times 2$ permanent $d_{i j} d_{k l}+d_{i l} d_{k j}$ corresponding to

$$
P=\left(\begin{array}{cc}
d_{i j} & d_{i l} \\
d_{k j} & d_{k l}
\end{array}\right) .
$$

Recall that $\operatorname{det}(A)=\sum_{\sigma \in S_{n}} \operatorname{Sgn}(\sigma) \Pi a_{i, \sigma(i)}$. There are $n$ ! terms in the expansion of the determinant. If a term does not contain either monomial $a_{i j} a_{k l}$ or $a_{i l} a_{k j}$, then the result of the action of the permanent $d_{i j} d_{k l}+d_{i l} d_{k j}$ on it will be zero. There are $(n-2)$ ! terms which contain the monomial $a_{i j} a_{k l}$ and $(n-2)$ ! terms which contain the monomial 
$a_{i l} a_{k j}$. So assume we have a permutation $\sigma_{1}$ of $n$ objects having $a_{i j}$ and $a_{k l}$, respectively, in its $i$ th and $k$ th place. Corresponding to $\sigma_{1}$, we also have a permutation $\sigma_{2}=\tau \sigma_{1}$, where $\tau=(j, l)$ is a transposition and $\operatorname{sgn}\left(\sigma_{2}\right)=\operatorname{sgn}\left(\tau \sigma_{1}\right)=-\operatorname{sgn}\left(\sigma_{1}\right)$. Thus, corresponding to each positive term in the determinant which contains monomial $a_{i j} a_{k l}$ or $a_{i l} a_{k j}$, we have the same term with the negative sign; thus, the resulting action of the permanent $d_{i j} d_{k l}+d_{i l} d_{k j}$ on $\operatorname{det}(A)$ is zero.

Definition 2.3. Let $A=\left(a_{i j}\right)$ and $D=\left(d_{i j}\right)$ be two generic matrices with entries in the polynomial ring $R=\mathrm{k}\left[a_{i j}\right]$ and in the ring of differential operators $S=\mathrm{k}\left[d_{i j}\right]$, respectively. Let $\left\{\mathcal{P}_{A}\right\},\left\{\mathcal{M}_{A}\right\},\left\{\mathcal{P}_{D}\right\}$ and $\left\{\mathcal{M}_{D}\right\}$ denote the set of all $2 \times 2$ permanents and the set of all $2 \times 2$ minors of $A$ and $D$, respectively. And, let $\mathcal{P}_{A}, \mathcal{M}_{A}=M_{2}(A), \mathcal{P}_{D}$ and $\mathcal{M}_{D}=M_{2}(D)$ denote the vector spaces they span, respectively.

Corollary 2.4. Each $2 \times 2$ permanent of $D$ annihilates $\mathcal{M}_{A}$.

Proof. By Lemma 2.2, $\mathcal{P}_{D} \circ \operatorname{det}(A)=0$. Let $F=\operatorname{det}(A)$. We have

$$
(\operatorname{Ann} F)_{2}=\left(\operatorname{Ann}\left(S_{n-2} \circ F\right)\right)_{2} \text {. }
$$

Hence,

$$
\mathcal{P}_{D} \circ \operatorname{det}(A)=0 \Longleftrightarrow \mathcal{P}_{D} \circ S_{n-2}(\operatorname{det}(A))=0 \Longleftrightarrow \mathcal{P}_{D} \circ \mathcal{M}_{A}=0 .
$$

We also know that the square of an element, or any product of two or more elements of the same row or column of $D$ annihilates $\operatorname{det}(A)$.

Definition 2.5. A monomial in the $n^{2}$ variables of the ring $S=\mathrm{k}\left[d_{i j}\right]$ is acceptable, if it is square free and has no two variables from the same row or column of $D$. A polynomial is acceptable if it can be written as the sum of acceptable monomials. A monomial is unacceptable if it does not divide any term of the determinant of the matrix $D$. A polynomial is unacceptable if it can be written as the sum of some unacceptable monomials. We denote the space of all unacceptable polynomials of degree 2 by $\mathcal{U}_{D}$.

We denote by $\langle X\rangle$ the k-vector space span of the set $X$.

Lemma 2.6. $\mathcal{P}_{D} \oplus \mathcal{M}_{D}=\langle$ degree 2 acceptable polynomials in $S\rangle$. 
Proof. Let $d_{i j} d_{k l}$ be an arbitrary acceptable monomial of degree 2 . Since $\operatorname{char}(k) \neq 2$, we have:

$$
d_{i j} d_{k l}=1 / 2\left(\left(d_{i j} d_{k l}-d_{i j} d_{k l}\right)+\left(d_{i j} d_{k l}+d_{i j} d_{k l}\right)\right) .
$$

By equation (2.1),

$$
\operatorname{dim} \mathcal{P}_{D}=\operatorname{dim} \mathcal{M}_{D}=\left(\begin{array}{l}
n \\
2
\end{array}\right)^{2}
$$

Let $\Psi=\langle$ degree 2 acceptable polynomials in $S\rangle$. Then

$$
\operatorname{dim} \Psi=\operatorname{dim} S_{2}-\operatorname{dim} \mathcal{U}_{D}=\left(\begin{array}{c}
n^{2}+1 \\
2
\end{array}\right)-\left(n^{2}+\left(\begin{array}{l}
n \\
2
\end{array}\right)(2 n)\right) .
$$

So we have

$$
\begin{aligned}
\operatorname{dim}\left(\mathcal{P}_{D}+\mathcal{M}_{D}\right) & =\left(\begin{array}{c}
n^{2}+1 \\
2
\end{array}\right)-\left(n^{2}+\left(\begin{array}{l}
n \\
2
\end{array}\right)(2 n)\right) \\
& =\operatorname{dim} \mathcal{P}_{D}+\operatorname{dim} \mathcal{M}_{D}
\end{aligned}
$$

Hence, $\mathcal{P}_{D} \cap \mathcal{M}_{D}=0$.

We have shown that $\left(\mathcal{P}_{D}+\mathcal{U}_{D}\right) \circ \mathcal{M}_{A}=0$ so $\mathcal{P}_{D}+\mathcal{U}_{D} \subset \operatorname{Ann}\left(\mathcal{M}_{A}\right)$. Then equation (1.2) implies

$$
\operatorname{dim}_{\mathrm{k}}\left(\operatorname{Ann}\left(\mathcal{M}_{A}\right)\right)_{2}=\operatorname{dim}_{\mathrm{k}} S_{2}-\operatorname{dim}_{\mathrm{k}} \mathcal{M}_{A} .
$$

Lemma 2.7. Ann $\left(\mathcal{M}_{A}\right) \cap S_{2}=\mathcal{P}_{D}+\mathcal{U}_{D}$

Proof. By Lemma 2.6, we have $\mathcal{P}_{D}+\mathcal{M}_{D}$ is complementary to $\mathcal{U}_{D}$. So we have

$$
\operatorname{dim}\left(\left(\operatorname{Ann}\left(\mathcal{M}_{A}\right)\right)_{2}\right)=\operatorname{dim} S_{2}-\operatorname{dim} \mathcal{M}_{A}=\operatorname{dim} \mathcal{P}_{D}+\mathcal{U}_{D}
$$

Notation. We define the homomorphism $\xi: R \rightarrow S$ by setting $\xi\left(a_{i j}\right)=d_{i j}$; for a monomial $v \in R$, we denote by $\widehat{v}=\xi(v)$ the corresponding monomial of $S$. 
Remark 2.8. Let $f=\sum_{i=1}^{i=k} \alpha_{i} v_{i} \in R_{n}$ with $\alpha_{i} \in \mathrm{k}$ and with $v_{i}$ 's linearly independent monomials. Then we will have:

$$
\operatorname{Ann}(f) \cap S_{n}=\left\langle\alpha_{j} \widehat{v_{1}}-\alpha_{1} \widehat{v_{j}},\left\langle v_{1}, \ldots, v_{k}\right\rangle^{\perp}\right\rangle,
$$

where $\left\langle v_{1}, \ldots, v_{k}\right\rangle^{\perp}=\operatorname{Ann}\left(\left\langle v_{1}, \ldots, v_{k}\right\rangle\right) \cap S_{n}$.

\section{Lemma 2.9.}

$$
\left(\mathcal{P}_{D}+\mathcal{U}_{D}\right)_{k} \subset \operatorname{Ann}\left(M_{k}(A)\right) \cap S_{k}
$$

Proof. We have:

(1) $\mathcal{P}_{D} \circ \operatorname{det}(A)=0 \Leftrightarrow \mathcal{P}_{D} \circ S_{n-2}(\operatorname{det}(A))=0 \Leftrightarrow \mathcal{P}_{D} \circ \mathcal{M}_{A}=0$.

(2) $(\operatorname{Ann}(\operatorname{det}(A))) \cap S_{2}=\mathcal{P}_{D}+\mathcal{U}_{D} \Rightarrow S_{k-2}\left(\mathcal{P}_{D}+\mathcal{U}_{D}\right) \circ\left(S_{n-k} \circ\right.$ $\operatorname{det}(A))=0$.

$$
\begin{aligned}
& \Longrightarrow S_{k-2}\left(\mathcal{P}_{D}+\mathcal{U}_{D}\right) \circ M_{k}(A)=0 . \\
& \Longrightarrow\left(\mathcal{P}_{D}+\mathcal{U}_{D}\right)_{k} \circ M_{k}(A)=0 \quad(\text { by Remark 1.4). }
\end{aligned}
$$

So equation (2.4) holds.

Proposition 2.10. For a generic $n \times n$ matrix $A$ with $n \geq 2$, we have

$$
\left(\mathcal{P}_{D}+\mathcal{U}_{D}\right)_{n}=\operatorname{Ann}(\operatorname{det}(A)) \cap S_{n}
$$

Proof. Using equation (2.4), we only need to show

$$
\left(\mathcal{P}_{D}+\mathcal{U}_{D}\right)_{n} \supset \operatorname{Ann}(\operatorname{det}(A)) \cap S_{n} .
$$

We use induction on $n$. For $n=2$, the equality is easy to see. Next we verify that the proposition holds for the case $n=3$. We need to see that the space of $2 \times 2$ permanents of $D$ generates $\operatorname{Ann}(\operatorname{det}(A))_{3} / \mathcal{U}_{D}$, i.e., Ann $\left(M_{3}(A)\right)_{3} / \mathcal{U}_{D}$. Corresponding to each term in the determinant, there is a permutation of three objects $\sigma$ such that we can write the term as $a_{1 \sigma(1)} a_{2 \sigma(2)} a_{3 \sigma(3)}$. Consider the degree three binomial $b=a_{1 \sigma(1)} a_{2 \sigma(2)} a_{3 \sigma(3)}-a_{1 \tau(1)} a_{2 \tau(2)} a_{3 \tau(3)}$, where $\tau \neq \sigma$. Without loss of generality, we can assume that $\sigma$ is the identity, so we consider the binomial $b=a_{11} a_{22} a_{33}-a_{1 \tau(1)} a_{2 \tau(2)} a_{3 \tau(3)}$. If these two monomials have a common variable, i.e., $\tau(i)=i$ for some $i=1,2,3$, then the binomial will be of the form $b=a_{i i}\left(a_{j j} a_{k k}-a_{j k} a_{k j}\right), 1 \leq i, j, k, l \leq 3$, so we will have $b=a_{i i} M_{i i}$ and, as we have shown previously, $P_{i i}=$ 
$d_{j j} d_{k k}+d_{j k} d_{k j}$ annihilates it. Assume that the monomials $a_{11} a_{22} a_{33}$ and $a_{1 \tau(1)} a_{2 \tau(2)} a_{3 \tau(3)}$ do not have any common factor. We can add and subtract another term $a_{1 \beta(1)} a_{2 \beta(2)} a_{3 \beta(3)}$, where $\beta$ is a permutation, such that it will have one common factor with $a_{11} a_{22} a_{33}$ and one common factor with $a_{1 \tau(1)} a_{2 \tau(2)} a_{3 \tau(3)}$. By reindexing, we can take $\beta(1)=\tau(1), \beta(2)=2$ and then we can determine $\beta(3)$ according to the other two choices. Then by factoring we get a binomial of the form $a_{i j} M_{i j}+a_{k l} M_{k l}$, where the first term can be annihilated by the permanent of the matrix $D$ corresponding to $d_{i j}$ and the second term can be annihilated by the permanent of the matrix $D$ corresponding to the element $d_{k l}$. So, by equation (2.3), we are done. For example, if we have the binomial $a_{11} a_{22} a_{33}-a_{13} a_{21} a_{32}$, we can add and subtract the term $a_{11} a_{23} a_{32}$ which has one common factor with $a_{11} a_{22} a_{33}$ and one common factor with $a_{13} a_{21} a_{32}$ so we will get $a_{11}\left(a_{22} a_{33}-a_{23} a_{32}\right)+a_{32}\left(a_{11} a_{23}-a_{13} a_{21}\right)$ which is $a_{11} M_{11}+a_{32} M_{32}$. And, as we have shown before, it can be annihilated by the space of $2 \times 2$ permanents. So, by equation (2.3), we are done.

When $n$ is larger than 3 , then by the induction assumption, we can assume that the proposition holds for all $k \leq n-1$. By Remark 2.8, it is enough to show that if $b$ is a binomial in $\operatorname{Ann}(\operatorname{det}(A)) \cap S_{n}$ of the form given by equation 2.3, then $b \in\left(\mathcal{P}_{D}+\mathcal{U}_{D}\right)_{n}$. Assume $b=b_{1}+b_{2}$ is of degree $n$. If the two terms, $b_{1}$ and $b_{2}$ are monomials in $S$ and have a common factor $l$, i.e., $b_{1}=l a_{1}$ and $b_{2}=l a_{2}$, then $b=l\left(a_{1}+a_{2}\right)$ where $a_{1}$ and $a_{2}$ are of degree at most $n-1$. So, by the induction assumption, the proposition holds for the binomial $a_{1}+a_{2}$, i.e., $a_{1}+a_{2} \in\left(\mathcal{P}_{D}+\mathcal{U}_{D}\right)_{n-1}$. Hence, we have

$$
b=l\left(a_{1}+a_{2}\right) \in l\left(\mathcal{P}_{D}+\mathcal{U}_{D}\right)_{n-1} \subset\left(\mathcal{P}_{D}+\mathcal{U}_{D}\right)_{n} .
$$

If the two terms, $b_{1}$ and $b_{2}$, do not have any common factor, then with the same method as above we can rewrite the binomial $b$ by adding and subtracting a term of the determinant, $m$ of degree $n$, which has a common factor $m_{1}$ with $b_{1}$ and a common factor $m_{2}$ with $b_{2}$. Then we will have

$$
b_{1}+b_{2}=b_{1}+m+b_{2}-m=m_{1}\left(c_{1}+m^{\prime}\right)+m_{2}\left(c_{2}-m^{\prime \prime}\right),
$$

where $b_{1}=m_{1} c_{1}, m=m_{1} m^{\prime}=m_{2} m^{\prime \prime}$ and $b_{2}=m_{2} c_{2}$. Since $c_{1}+m^{\prime}$ and $c_{2}-m^{\prime \prime}$ are of degree at most $n-1$, the induction assumption 
yields

$$
b_{1}+b_{2}=m_{1}\left(c_{1}+m^{\prime}\right)+m_{2}\left(c_{2}-m^{\prime \prime}\right) \in\left(\mathcal{P}_{D}+\mathcal{U}_{D}\right)_{n} .
$$

This completes the induction step and hence the proof of the proposition.

Corollary 2.11. For a generic $n \times n$ matrix $A$ and each integer $k$, $1 \leq k \leq n$, we have

$$
\left(\mathcal{P}_{D}+\mathcal{U}_{D}\right)_{k}=\operatorname{Ann}(\operatorname{det}(A)) \cap S_{k}=\left(\operatorname{Ann}\left(M_{k}(A)\right)\right)_{k} .
$$

We also have $\left(\mathcal{U}_{D}\right)_{n+1}=S_{n+1}$.

Proof. Using equation (2.4) we only need to show that

$$
\operatorname{Ann}(\operatorname{det}(A)) \cap S_{k} \subset\left(\mathcal{P}_{D}+\mathcal{U}_{D}\right)_{k} .
$$

By Lemma 1.3 and Remark 1.4, we have

$$
(\operatorname{Ann}(\operatorname{det}(A)))_{k}=\left(\operatorname{Ann}\left(S_{n-k} \circ(\operatorname{det}(A))\right)\right)_{k}=\left(\operatorname{Ann}\left(M_{k}(A)\right)\right)_{k} .
$$

If we label the $k \times k$ minors of $A$ by $f_{1}, \ldots, f_{s}$ we have

$$
\left(\operatorname{Ann}\left(M_{k}(A)\right)\right)_{k}=\operatorname{Ann}\left(\left\langle f_{1}, \ldots, f_{s}\right\rangle\right)_{k}=\left(\bigcap_{i=1}^{i=s}\left(\operatorname{Ann}\left(f_{i}\right)\right)\right)_{k} .
$$

For each $f_{i}$ we denote the ring of variables of $f_{i}$ by $R^{i}$ and the ring of differential operators by $S^{i}$. Then, by Proposition 2.10, we have

$$
\left(\mathcal{P}_{D}^{i}+\mathcal{U}_{D}^{i}\right)_{k}=\operatorname{Ann}\left(f_{i}\right) \cap S_{k}^{i} .
$$

Hence,

$$
\operatorname{Ann}(\operatorname{det}(A)) \cap S_{k} \subset\left(\mathcal{P}_{D}+\mathcal{U}_{D}\right)_{k}
$$

Finally, every monomial of degree larger than $n$ will be unacceptable, so is in $\mathcal{U}_{D}$, as the unacceptable monomials are generated in degree 2 . So we have $\left(\mathcal{U}_{D}\right)_{n+1}=S_{n+1}$.

Theorem 2.12. Let $A$ be a generic $n \times n$ matrix. Then the apolar ideal $\operatorname{Ann}(\operatorname{det}(A)) \subset S$ is the ideal $\left(\mathcal{P}_{D}+\mathcal{U}_{D}\right)$ and is generated in degree 2.

Proof. This follows directly from Proposition 2.10 and Corollary 2.11. 
Theorem 2.13. Let $A$ be a generic $n \times n$ matrix. Then the apolar ideal Ann $(\operatorname{Per}(A)) \subset S$ to $\operatorname{Per}(A) \in R$ is the ideal $\left(\mathcal{M}_{D}+\mathcal{U}_{D}\right)$, generated in degree 2 .

Proof. The proof follows directly from the proof of Proposition 2.10 and Corollary 2.11, by interchanging the determinants and the permanents.

Corollary 2.14. Let $A=\left(a_{i j}\right)$ be an $m \times n$ matrix where $n \geq m$. Let $N$ denote the space generated by all $m \times m$ minors of $A$. Then Ann $(N)$ is generated in degree 2 by all $2 \times 2$ permanents of $A$ and the degree two unacceptable monomials.

Proof. Let $s=\left(\begin{array}{c}n \\ m\end{array}\right)$, and let $f_{1}, \ldots, f_{s}$ denote the $m \times m$ minors of A. We have

$$
\operatorname{Ann}(N)=\operatorname{Ann}\left(\left\langle f_{1}, \ldots, f_{s}\right\rangle\right)=\bigcap_{i=1}^{i=s}\left(\operatorname{Ann}\left(f_{i}\right)\right) .
$$

Let $R^{i}$ denote the ring of variables of $f_{i}$. Hence, by Theorem 2.12, we have $\operatorname{Ann}\left(f_{i}\right) \cap S^{i}$ is generated in degree 2. So we have $\operatorname{Ann}(N)$ is also generated in degree 2 .

\section{Application to the ranks of the determinant and the permanent.}

Notation. Let $F \in R=\mathrm{k}\left[a_{i j}\right]$ be a homogeneous form of degree $d$. A presentation

$$
F=l_{1}^{d}+\cdots+l_{s}^{d} \quad \text { with } l_{i} \in R_{1} .
$$

is called a Waring decomposition of length $s$ of the polynomial $F$. The minimal number $s$ that satisfies equation (3.1) is called the rank of $F$.

The apolarity action of $S=\mathrm{k}\left[d_{i j}\right]$ on $R$ defines $S$ as a natural coordinate ring on the projective space $\mathbf{P}\left(R_{1}\right)$ of one-dimensional subspaces of $R_{1}$ and vice versa. A finite subscheme $\Gamma \subset \mathbf{P}\left(R_{1}\right)$ is apolar to $F$ if the homogeneous ideal $I_{\Gamma} \subset S$ is contained in $\operatorname{Ann}(F)$ $([13,19])$. 
Remark 3.1. ([13, Definition 5.66], $[\mathbf{1 9}])$. Let $\Gamma=\left\{\left[l_{1}\right], \ldots,\left[l_{s}\right]\right\}$ be a collection of $s$ distinct points in $\mathbf{P}\left(R_{1}\right)$. Then

$$
F=c_{1} l_{1}^{d}+\cdots+c_{s} l_{s}^{d} \quad \text { with } c_{i} \in \mathrm{k}
$$

if and only if

$$
I_{\Gamma} \subset \operatorname{Ann}(F) \subset S .
$$

Definition 3.2. We have the following ranks ([3], [13, Definition 5.66], [19]). Here $\Gamma$ is a punctual scheme (possibly not smooth), and the degree of $\Gamma$ is the number of points (counting multiplicities) in $\Gamma$.

(a) the rank $r(F)$ :

$$
r(F)=\min \left\{\operatorname{deg} \Gamma \mid \Gamma \subset \mathbf{P}\left(R_{1}\right) \text { smooth }, \operatorname{dim} \Gamma=0, I_{\Gamma} \subset \operatorname{Ann}(F)\right\} .
$$

Note that when $\Gamma$ is smooth, it is the set of points in Remark 3.1 ([13, page 135]).

(b) the smoothable rank $\operatorname{sr}(F)$ :

$$
\begin{gathered}
\operatorname{sr}(F)=\min \left\{\operatorname{deg} \Gamma \mid \Gamma \subset \mathbf{P}\left(R_{1}\right)\right. \text { smoothable, } \\
\left.\operatorname{dim} \Gamma=0, I_{\Gamma} \subset \operatorname{Ann}(F)\right\} .
\end{gathered}
$$

Note that, for the smoothable rank, one considers the smoothable schemes that are the schemes which are the limits of smooth schemes of $s$ simple points ([13, Definition 5.66]).

(c) the cactus rank (scheme length in [13, Definition 5.1, page 135]) $\operatorname{cr}(F)$ :

$$
\operatorname{cr}(F)=\min \left\{\operatorname{deg} \Gamma \mid \Gamma \subset \mathbf{P}\left(R_{1}\right), \operatorname{dim} \Gamma=0, I_{\Gamma} \subset \operatorname{Ann}(F)\right\} .
$$

(d) the differential rank (Sylvester's catalecticant or apolarity bound) is the maximal dimension of a homogeneous component of $S / \operatorname{Ann}(F)$ :

$$
l_{\text {diff }}(F)=\max _{i \in \mathbb{N}_{0}}\left\{(H(S / \operatorname{Ann}(F)))_{i}\right\} .
$$

Note that we give a lower bound for the cactus rank of the determinant and permanent of the generic matrix. We do not have further information on the smoothable rank of the generic determinant or permanent. It is still open to find good bounds for the smoothable rank. The work of Bernardi and Ranestad [3] in the case of generic forms of 
a given degree and number of variables shows that the cactus rank and smoothable rank can be very different.

Proposition 3.3. ([13, Proposition $6.7 \mathrm{C}]$ ). The above ranks satisfy

$$
l_{\text {diff }}(F) \leq c r(F) \leq s r(F) \leq r(F) .
$$

Proposition 3.4. (Ranestad and Schreyer) ([19, Proposition 1]). If the ideal of $\operatorname{Ann}(F)$ is generated in degree $d$ and $\Gamma \subset \boldsymbol{P}\left(T_{1}\right)$ is a finite (punctual) apolar subscheme to $F$, then

$$
\operatorname{deg} \Gamma \geq \frac{1}{d} \operatorname{deg}(\operatorname{Ann}(F))
$$

where $\operatorname{deg}(\operatorname{Ann}(F))=\operatorname{dim}(S / \operatorname{Ann}(F))$ is the length of the zerodimensional scheme defined by $\operatorname{An}(F)$.

Remark 3.5. The Ranestad-Schreyer proposition is true for arbitrary characteristic: the argument depends on Bézout's theorem, which is true for $k$ algebraically closed (see [10, page 113]); and none of the degrees involved in the proof changes as one extends from an infinite field $\mathrm{k}$ to its algebraic closure.

Since we have shown that, for $F=\operatorname{det}(A)$ or $F=\operatorname{Per}(A)$, we have $d=2$, we can use Proposition 3.4 to give a lower bound for the above ranks of $F$.

Theorem 3.6. Let $F$ be the determinant or permanent of a generic $n \times n$ matrix $A$. We have

$$
\frac{1}{2}\left(\begin{array}{c}
2 n \\
n
\end{array}\right) \leq c r(F) \leq s r(F) \leq r(F) .
$$

Proof. By Theorems 2.12 and 2.13, Propositions 3.4 and 3.3 and equations (2.1) and (2.2), we have for an apolar punctual scheme $\Gamma$,

$$
\operatorname{deg} \Gamma \geq \frac{1}{d} \operatorname{deg}\left(\operatorname{Ann}(F)=\frac{1}{2} \sum_{k=0}^{k=n}\left(\begin{array}{l}
n \\
k
\end{array}\right)^{2}=\frac{1}{2}\left(\begin{array}{c}
2 n \\
n
\end{array}\right) .\right.
$$


Notation [15]. Let $\Phi \in S^{d} \mathbb{C}^{n}$ be a polynomial. We can polarize $\Phi$, consider it as a multilinear form $\widetilde{\Phi}$, where $\Phi(x)=\widetilde{\Phi}(x, \ldots, x)$, and consider the linear map $\Phi_{s, d-s}: S^{s} \mathbb{C}^{n *} \rightarrow S^{d-s} \mathbb{C}^{n}$ where $\Phi_{s, d-s}\left(x_{1}, \ldots, x_{s}\right)\left(y_{1}, \ldots, y_{d-s}\right)=\widetilde{\Phi}\left(x_{1}, \ldots, x_{s}, y_{1}, \ldots, y_{d-s}\right)$. Define

$$
\operatorname{Zeros}(\Phi)=\left\{[x] \in \mathbb{P C}^{n *} \mid \Phi(x)=0\right\} \subset \mathbb{P} \mathbb{C}^{n *} .
$$

Let $x_{1}, \ldots, x_{n}$ be linear coordinates on $\mathbb{C}^{n *}$, and define

$$
\Sigma_{s}(\Phi):=\left\{[x] \in \operatorname{Zeros}(\Phi) \mid \frac{\partial^{I} \Phi}{\partial x^{I}}(x)=0, \forall I, \text { such that }|I| \leq s\right\} .
$$

In this notation, $\Phi_{s, d-s}$ is the map from $S_{s} \rightarrow R_{n-s}$ taking $h$ to $h \circ \Phi$; hence, its rank is $H\left(\mathfrak{A}_{A}\right)_{s}$.

In the following theorem, we use the convention that $\operatorname{dim} \emptyset=-1$.

Theorem 3.7. (Landsberg-Teitler) ([15, Theorem 1.3]). Let $\Phi \in$ $S^{d} \mathbb{C}^{n}$, and let $1 \leq s \leq d$. Then

$$
\operatorname{rank}(\Phi) \geq \operatorname{rank} \Phi_{s, d-s}+\operatorname{dim} \Sigma_{s}(\Phi)+1 .
$$

Remark 3.8 (Teitler). If we define $\Sigma_{s}(\Phi)$ to be a subset of affine rather than projective space, then the above theorem does not need +1 at the end and does not need the statement that the dimension of the empty set is -1 .

Applying this theorem to the determinant yields:

Corollary 3.9. ([15]). For $\operatorname{char}(\mathrm{k})=0$,

$$
r\left(\operatorname{det}_{n}\right) \geq\left(\begin{array}{c}
n \\
\lfloor n / 2\rfloor
\end{array}\right)^{2}+n^{2}-(\lfloor n / 2\rfloor+1)^{2} .
$$

Proposition 3.10. (Bernardi and Ranestad) ([3, Theorem 1]). Let $F \in R^{s}$ be a homogeneous form of degree $d$, and let $l$ be any linear form in $S_{1}^{s}$. Let $F_{l}$ be a dehomogenization of $F$ with respect to $l$. Denote by Diff $(F)$ the subspace of $S^{s}$ generated by the partials of $F$ of all orders. Then

$$
c r(F) \leq \operatorname{dim}_{\mathrm{k}} \operatorname{Diff}\left(F_{l}\right) .
$$


In [3] this is stated for $\mathbb{C}$, but the proof is valid for any field provided one uses contraction and the divided power ring. We thank Pedro Marques for pointing out that it is easy to show that the length of a polynomial is an upper bound for the length of any dehomogenization of that polynomial. So we have

$$
\operatorname{cr}(F) \leq \operatorname{dim}_{\mathrm{k}} \operatorname{Diff}(F)=\operatorname{deg}(\operatorname{Ann}(F))
$$

Proposition 3.11. For the monomial $m=x_{1}^{b_{1}} \cdots x_{n}^{b_{n}}$, where $1 \leq b_{1} \leq$ $\cdots \leq b_{n}$, we have

(a) $([7])$

$$
r\left(x_{1}^{b_{1}} \cdots x_{n}^{b_{n}}\right)=\prod_{i=2}^{i=n}\left(b_{i}+1\right) .
$$

(b) $([\mathbf{1 9}])$

$$
\operatorname{sr}\left(x_{1}^{b_{1}} \cdots x_{n}^{b_{n}}\right)=\operatorname{cr}\left(x_{1}^{b_{1}} \cdots x_{n}^{b_{n}}\right)=\prod_{i=1}^{i=n-1}\left(b_{i}+1\right) .
$$

(c) ([6]) Let $d=b_{1}+\cdots+b_{n}$, and $m=l_{1}^{d}+\cdots+l_{s}^{d}$ with $r(m)=s$. Let $I \subset S$ be the homogeneous ideal of functions vanishing on $Q=\left\{\left[l_{1}\right], \ldots,\left[l_{s}\right]\right\} \subset \boldsymbol{P}^{n-1}$. Then $I$ is a complete intersection of degrees $b_{2}+1, \ldots, b_{n}+1$ generated by

$$
y_{2}^{b_{2}+1}-\Phi_{1} y_{1}^{b_{1}+1}, \ldots, y_{n}^{b_{n}+1}-\Phi_{n} y_{1}^{b_{1}+1},
$$

for some homogeneous polynomials $\Phi_{i} \in S$ of degree $b_{i}-b_{1}$.

Example 3.12. Let $n=2$, and

$$
\begin{aligned}
A & =\left(\begin{array}{ll}
a & b \\
c & d
\end{array}\right), \\
\operatorname{det}(A) & =a d-b c \\
& =(a+d)^{2}-(a-d)^{2}+(b-c)^{2}-(b+c)^{2},
\end{aligned}
$$

so $r(\operatorname{det}(A))=4$. The corresponding Hilbert sequence for $n=2$ is $(1,4,1)$. We have $l_{\text {diff }}(\operatorname{det}(A))=4$. Using Theorem 3.6, we have:

$$
\operatorname{cr}(\operatorname{det}(A)) \geq \frac{1}{d} \operatorname{deg}(\operatorname{Ann}(\operatorname{det}(A)))=\frac{1}{2}(6)=3 .
$$

So the lower bound we obtain using Theorem 3.6 is 3 . 
Using Corollary 3.9 (Landsberg and Teitler) for $\mathrm{k}=\mathbb{C}$, we obtain

$$
r(\underset{2}{\operatorname{det}}) \geq\left(\begin{array}{c}
2 \\
\lfloor 2 / 2\rfloor
\end{array}\right)^{2}+2^{2}-(\lfloor 2 / 2\rfloor+1)^{2}=4+4-4=4 .
$$

On the other hand, we have

$$
\begin{aligned}
\operatorname{det}(A) & =a d-b c \\
& =1 / 4\left((a+d)^{2}-(a-d)^{2}\right)-1 / 4\left((b+c)^{2}-(b-c)^{2}\right) .
\end{aligned}
$$

Hence, for any field

$$
r(\operatorname{det}(A))=\operatorname{cr}(\operatorname{det}(A))=\operatorname{sr}(\operatorname{det}(A))=l_{\mathrm{diff}}(\operatorname{det}(A))=4 .
$$

Example 3.13. Let $n=3$, and let

$$
\begin{aligned}
A & =\left(\begin{array}{lll}
a & b & e \\
c & d & f \\
g & h & i
\end{array}\right), \\
\operatorname{det}(A) & =g(b f-d e)-h(a f-c e)+i(a d-b c) .
\end{aligned}
$$

Using Macaulay2 [11] for the calculations we obtain the Hilbert sequence $(1,9,9,1)$ and, by Theorem 3.6 , we have:

$$
\operatorname{cr}(\operatorname{det}(A)) \geq \frac{1}{d} \operatorname{deg}(\operatorname{Ann}(\operatorname{det}(A)))=\frac{1}{2}(20)=10 \text {. }
$$

So the lower bound we find using Theorem 3.6 is 10, which is greater than the $l_{\text {diff }}(\operatorname{det}(A))=9$, so it is a better lower bound than the differential length for the cactus and smoothable ranks introduced above.

Using Corollary 3.9, we have:

$$
r(\underset{3}{\operatorname{det}}) \geq\left(\begin{array}{c}
3 \\
\lfloor 3 / 2\rfloor
\end{array}\right)^{2}+3^{2}-(\lfloor 3 / 2\rfloor+1)^{2}=9+9-4=14 .
$$

On the other hand, for every $x, y$ and $z$, it is easy to see that $r(x y z) \leq 4$ :

$$
\begin{aligned}
x y z= & 1 / 24\left((x+y+z)^{3}+(x-y-z)^{3}\right. \\
& \left.-(x-y+z)^{3}-(x+y-z)^{3}\right) .
\end{aligned}
$$

Hence, $14 \leq r(\operatorname{det}(A)) \leq 24$. 
If $a=1$ in $\operatorname{det}(A)$, we have that the punctual scheme $\operatorname{Ann}\left(\operatorname{det} A_{a=1}\right)$ of degree 18 with Hilbert function $(1,8,8,1)$. So, by Proposition 3.10, we have:

$$
\operatorname{cr}(\operatorname{det}(A)) \leq 18 \text {. }
$$

Bernardi, Marques and Ranestad in [2, Theorem 1] have shown that the cactus rank of a generic homogeneous cubic form in 9 variables is 18 , which is an upper bound for the cactus rank of the determinant of a $3 \times 3$ generic matrix.

\section{Remark 3.14.}

(a) Using Stirling's formula, $n ! \sim \sqrt{2 \pi n}(n / e)^{n}$, we can approximate $\left(\begin{array}{c}2 n \\ n\end{array}\right)$ for large $n$ by $4^{n} / \sqrt{n \pi}$. Hence, for large $n$, Theorem 3.6 gives us a lower bound asymptotic to $4^{n} / 2 \sqrt{n \pi} \leq \operatorname{cr}(\operatorname{det}(A))$, and the Landsberg-Teitler formula gives us the lower bound $2 \cdot 4^{n} /(n \pi) \leq$ $r(\operatorname{det}(A))$. The Landsberg-Teitler lower bound for $r(\operatorname{det}(A))$ is also asymptotic to $l_{\text {diff }}(\operatorname{det}(A))=\left(\begin{array}{c}n \\ \lfloor n / 2\rfloor\end{array}\right)^{2}$, which is a lower bound for $\operatorname{cr}(\operatorname{det}(A))$. These are also lower bounds for the corresponding ranks of the permanent of a generic $n \times n$ matrix.

(b) By Proposition 3.11, an upper bound for the rank of the determinant and permanent of a generic $n \times n$ matrix is given by $(n !) 2^{n-1}$. This can be approximated for large $n$, using Stirling's formula, by $\sqrt{2 \pi n}(n / e)^{n}\left(2^{n-1}\right)$.

(c) By equation (3.2), an upper bound for the cactus rank of both the determinant and permanent of a generic $n \times n$ matrix is $\left(\begin{array}{c}2 n \\ n\end{array}\right)$, which is asymptotic to $4^{n} / \sqrt{n \pi}$.

By the Alexander and Hirschowitz theorem [1], the rank of a general form of degree $d$ in $m$ variables is as follows:

$$
s r_{\text {gen }}=r_{\text {gen }}=\left\lceil\frac{1}{m}\left(\begin{array}{c}
m+d-1 \\
d
\end{array}\right)\right\rceil,
$$

when $d>2$ and $(m, d) \neq(m, 2),(3,4),(4,4),(5,3),(5,4)$ (see [3, 22]). For $m=n^{2}$ and $d=n$, this is asymptotic to $c e^{n} n^{n-2.5}$ for some bounded $c$.

For a monomial $M$ in $m \geq 4$ variables in degree $d>1$, Teitler has shown that $r(M) \leq r_{\text {gen }}(m, 2)([\mathbf{2 2}$, Theorem 1]). The cactus rank of monomials is given by Ranestad and Schreyer (see Proposition 3.11, 
part b) and is maximized when the $n-1$ degrees are the same. The determinant and permanent have greater cactus rank than monomials of the same degree and number of variables.

In the following table, we give lower bounds for the ranks of the determinant and permanent of an $n \times n$ generic matrix.

TABLE 1. The determinant of the generic matrix.

\begin{tabular}{lcccccc}
\hline \multicolumn{1}{c}{$n$} & 2 & 3 & 4 & 5 & 6 & $n \gg 0$ \\
\hline $\begin{array}{l}\text { lower bound for } c r(\operatorname{det}(A)) \\
\text { by Theorem 3.6 }\end{array}$ & 3 & 10 & 35 & 126 & 462 & $4^{n} / 2 \sqrt{n \pi}$ \\
\hline $\begin{array}{l}\text { lower bound for } r(\operatorname{det}(A)) \\
\text { by Corollary 3.9 }\end{array}$ & 4 & 14 & 43 & 116 & 420 & $4^{n} /(2 n \pi)$ \\
\hline$l_{\text {diff }}(\operatorname{det}(A))$ & 4 & 9 & 36 & 100 & 400 & $\left(\begin{array}{c}n \\
\lfloor n / 2\rfloor\end{array}\right)$ \\
\hline
\end{tabular}

4. Annihilator of the Pfaffian and hafnian. In this section, we discuss the annihilator ideals of the Pfaffians and of the hafnians. We show that the annihilator ideal of the Pfaffian of a generic skew symmetric $2 n \times 2 n$ matrix and the annihilator ideal of the hafnian of generic symmetric $2 n \times 2 n$ matrix are both generated in degree 2 .

In the following discussion, we let $X_{m}^{s k}=\left(x_{i j}\right)$ with $x_{i j}=-x_{j i}$ be an $m \times m$ skew symmetric matrix of indeterminates in the polynomial $\operatorname{ring} R^{s k}=\mathrm{k}\left[x_{i j}\right]$. Let $Y_{m}^{s k}=\left(y_{i j}\right)$ with $y_{i j}=-y_{j i}$ be an $m \times m$ skew symmetric matrix of indeterminates in the ring of differential operators $S^{s k}=\mathrm{k}\left[y_{i j}\right]$. We denote the Pfaffian of the matrix $X_{m}^{s k}$ by $\operatorname{Pf}\left(X_{m}^{s k}\right)$. It is well known that for any odd number $m$ we have $\operatorname{det}\left(X_{m}^{s k}\right)=0$. It is also well known that the square of the Pfaffian is equal to the determinant of a skew symmetric matrix. So, in the following, we are going to consider the annihilator of the Pfaffian of generic $m \times m$ skew symmetric matrices, where $m=2 n$ is an even number. Recall that:

Notation. Let $F_{2 n} \subset S_{2 n}$ be the set of all permutations $\sigma$ satisfying the following conditions:

(1) $\sigma(1)<\sigma(3)<\cdots<\sigma(2 n-1)$,

(2) $\sigma(2 i-1)<\sigma(2 i)$ for all $1 \leq i \leq n$. 
- For a $2 n \times 2 n$ generic skew symmetric matrix $X^{s k}$, we denote by $\operatorname{Pf}\left(X^{s k}\right)$ the Pfaffian of $X^{s k}$ defined by

$$
\operatorname{Pf}\left(X^{s}\right)=\sum_{\sigma \in F_{2 n}} \operatorname{sgn}(\sigma) x_{\sigma(1) \sigma(2)} x_{\sigma(3) \sigma(4)} \cdots x_{\sigma(2 n-1) \sigma(2 n)}
$$

- ([14]) We denote by $H f\left(X^{s}\right)$ the hafnian of a generic symmetric $2 n \times 2 n$ matrix $X^{s}$ defined by

$$
H f\left(X^{s}\right)=\sum_{\sigma \in F_{2 n}} x_{\sigma(1) \sigma(2)} x_{\sigma(3) \sigma(4)} \cdots x_{\sigma(2 n-1) \sigma(2 n)}
$$

Let $J_{2 n}=\operatorname{Ann}\left(\operatorname{Pf}\left(X_{2 n}^{s k}\right)\right)$. We first give some examples and then some partial results concerning Ann $\left(P f\left(X_{2 n}^{s k}\right)\right)$. Using Macaulay2 for calculations we have the following results:

(a) Let $X_{2}$ be a generic skew symmetric $2 \times 2$ matrix, then we have $H\left(S^{s k} / J_{2}\right)=(1,1)$. And the maximum degree of the generators of the annihilator ideal $J_{2}$ is 2 . So, using the Ranestad-Schreyer proposition, we have:

$$
\operatorname{cr}\left(\operatorname{Pf}\left(X_{2}^{s k}\right)\right) \geq \frac{1}{d} \operatorname{deg}\left(\operatorname{Ann}\left(\operatorname{Pf}\left(X_{2}^{s k}\right)\right)\right)=\frac{1}{2}(2)=1,
$$

which is the same as the differential length in this case. Evidently, in this case, $r\left(\operatorname{Pf}\left(X_{2}^{s k}\right)=1\right.$, so we have

$$
r\left(\operatorname{Pf}\left(X_{2}^{s k}\right)\right)=\operatorname{cr}\left(\operatorname{Pf}\left(X_{2}^{s k}\right)\right)=\operatorname{sr}\left(\operatorname{Pf}\left(X_{2}^{s k}\right)\right)=l_{\mathrm{diff}}\left(\operatorname{Pf}\left(X_{2}^{s k}\right)\right)=1 .
$$

(b) Let $X_{4}$ be a generic skew symmetric $4 \times 4$ matrix. Using Macaulay 2 for calculations, we have $H\left(S^{s k} / J_{4}\right)=(1,6,1)$, and the maximum degree of the generators of the annihilator ideal $J_{4}$ is 2 . Using the Ranestad-Schreyer proposition, we have:

$$
\operatorname{cr}\left(\operatorname{Pf}\left(X_{4}^{s k}\right)\right) \geq \frac{1}{d} \operatorname{deg}\left(\operatorname{Ann}\left(\operatorname{Pf}\left(X_{4}^{s k}\right)\right)\right)=\frac{1}{2}(8)=4,
$$

which is less than $l_{\text {diff }}=6$.

(c) Let $X_{6}$ be a generic skew symmetric $6 \times 6$ matrix. Using Macaulay2 for calculations, we have $H\left(S^{s k} / J_{6}\right)=(1,15,15,1)$, and the maximum degree of the generators of the annihilator ideal $J_{6}$ is 2 . Using the Ranestad-Schreyer proposition, we have:

$$
\operatorname{cr}\left(\operatorname{Pf}\left(X_{6}^{s k}\right)\right) \geq \frac{1}{d} \operatorname{deg}\left(\operatorname{Ann}\left(\operatorname{Pf}\left(X_{6}^{s k}\right)\right)\right)=\frac{1}{2}(32)=16
$$


which is larger than $l_{\text {diff }}=15$.

(d) Let $X_{8}$ be a generic skew symmetric $8 \times 8$ matrix. Using Macaulay2 for calculations, we have $H\left(S^{s k} / J_{8}\right)=(1,28,70,28,1)$, and the maximum degree of the generators of the annihilator ideal $J_{8}$ is 2 . From the Ranestad-Schreyer proposition, we have:

$$
\operatorname{cr}\left(P f\left(X_{8}^{s k}\right)\right) \geq \frac{1}{d} \operatorname{deg}\left(\operatorname{Ann}\left(P f\left(X_{8}^{s k}\right)\right)\right)=\frac{1}{2}(128)=64,
$$

which is less than $l_{\text {diff }}=70$.

(e) Let $X_{10}$ be a generic skew symmetric $10 \times 10$ matrix. Using Macaulay2 for calculations, we have

$$
H\left(S^{s k} / J_{10}\right)=(1,45,210,210,45,1) .
$$

The maximum degree of the generators of the annihilator ideal $J_{10}$ is 2. From the Ranestad-Schreyer proposition, we have:

$$
c r\left(P f\left(X_{10}^{s k}\right)\right) \geq \frac{1}{d} \operatorname{deg}\left(\operatorname{Ann}\left(P f\left(X_{10}^{s k}\right)\right)\right)=\frac{1}{2}(512)=256,
$$

which is larger than $l_{\text {diff }}=210$.

Remark 4.1. The Hilbert sequence $H(\mathfrak{A})$ for the apolar algebra $\mathfrak{A}$ of the Pfaffian of a generic $2 n \times 2 n$ matrix is given by $\left(\begin{array}{c}2 n \\ 2 i\end{array}\right)$, and we have $\sum_{i=0}^{i=n}\left(\begin{array}{c}2 n \\ 2 i\end{array}\right)=2^{2 n-1}$.

Definition 4.2. A $2 t$-Pfaffian minor of a skew symmetric matrix $X$ is a Pfaffian of a submatrix of $X$ consisting of rows and columns indexed by $i_{1}, i_{2}, \ldots, i_{2 t}$ for some $i_{1}<i_{2}<\cdots<i_{2 t}$.

The number of $2 t$-Pfaffian minors of a $2 n \times 2 n$ skew symmetric matrix is clearly $\left(\begin{array}{c}2 n \\ 2 t\end{array}\right)$. We denote by $\left\{P_{2 t}\left(X^{s k}\right)\right\}$ the set of the $2 t$ Pfaffians of $X^{s k}$. Furthermore, we denote by $P_{2 t}\left(X^{s k}\right)$ the vector space generated by $\left\{P_{2 t}\left(X^{s k}\right)\right\}$ in $R_{t}^{s k}$, and we denote by $\left(P_{2 t}\left(X^{s k}\right)\right)$ the ideal generated by $\left\{P_{2 t}\left(X^{s k}\right)\right\}$ in $R^{s k}$. Let $\tau$ be the lexicographic term order on $R^{s k}=\mathrm{k}\left[x_{i j}\right]$ induced by the following order on the indeterminates:

$$
x_{1,2 n} \geq x_{1,2 n-1} \geq \cdots \geq x_{1,2} \geq x_{2,2 n} \geq x_{2,2 n-1} \geq \cdots \geq x_{2 n-1,2 n} .
$$


Theorem 4.3. (Herzog and Trung [12, Theorem 4.1]). The set $\left\{P_{2 t}(X)\right\}$ of the $2 t$-Pfaffians of the matrix $X^{s k}$ is a Gröbner basis of the ideal $\left(P_{2 t}(X)\right)$ with respect to $\tau$.

Corollary 4.4. The dimension of the space of $2 t \times 2 t$ Pfaffians of a $2 n \times 2 n$ generic skew symmetric matrix $X^{\text {sk }}$ is $\left(\begin{array}{c}2 n \\ 2 t\end{array}\right)$. So we have

$$
\operatorname{dim}\left(S^{s k} / \operatorname{Ann}\left(\operatorname{Pf}\left(X^{s k}\right)\right)\right)=2^{2 n-1} .
$$

Proof. The proof follows directly from Theorem 4.3 and the combinatorial identity:

$$
\sum_{t=0}^{t=n}\left(\begin{array}{c}
2 n \\
2 t
\end{array}\right)=2^{2 n-1} .
$$

This identity is easy to show, e.g., it follows immediately by evaluating at $x=1$ and $x=-1$ the binomial expansion of $(x+1)^{2 n}$.

The examples strongly suggest that the apolar ideal of the Pfaffian is generated in degree 2 . In the remaining part of this section we prove that this is always the case.

Definition 4.5. Let $W$ be the vector subspace of $S^{s k}$ spanned by degree 2 elements of type (a), (b) and (c) defined as follows:

(a) square of each element of $Y^{s k}$. The number of these monomials is $2 n^{2}-n$.

(b) The product of each element of $Y^{s k}$ with another element in the same row or column of the matrix $Y^{s k}$. The number of these monomials is $\left(2 n^{2}-n\right)(2 n-2)$.

(c) Given any $4 \times 4$ submatrix of $X^{s k}$ of the rows and columns $i_{1}, i_{2}, i_{3}$ and $i_{4}$,

$$
Q=\left(\begin{array}{cccc}
0 & x_{i_{1} i_{2}} & x_{i_{1} i_{3}} & x_{i_{1} i_{4}} \\
-x_{i_{1} i_{2}} & 0 & x_{i_{2} i_{3}} & x_{i_{2} i_{4}} \\
-x_{i_{1} i_{3}} & -x_{i_{2} i_{3}} & 0 & x_{i_{3} i_{4}} \\
-x_{i_{1} i_{4}} & -x_{i_{2} i_{4}} & -x_{i_{3} i_{4}} & 0
\end{array}\right),
$$

we have $\operatorname{Pf}(Q)=x_{i_{1} i_{2}} x_{i_{3} i_{4}}-x_{i_{1} i_{3}} x_{i_{2} i_{4}}+x_{i_{1} i_{4}} x_{i_{2} i_{3}}$.

Corresponding to $\operatorname{Pf}(Q)$, we have three binomials which annihilate $P f(Q)$ and hence annihilate $\operatorname{Pf}\left(X^{s k}\right)$. These binomials are: $y_{i_{1} i_{2}} y_{i_{3} i_{4}}+$ 
$y_{i_{1} i_{3}} y_{i_{2} i_{4}}, y_{i_{1} i_{2}} y_{i_{3} i_{4}}-y_{i_{1} i_{4}} y_{i_{2} i_{3}}$ and $y_{i_{1} i_{3}} y_{i_{2} i_{4}}+y_{i_{1} i_{4}} y_{i_{2} i_{3}}$. However, these three binomials are not linearly independent, and we can write one of them as the sum of the other two binomials. So, corresponding to each $4 \times 4$ Pfaffian, we have two linearly independent binomials in the annihilator ideal and, using Theorem 4.3, the number of these binomials is $2 \cdot\left(\begin{array}{c}2 n \\ 4\end{array}\right)$.

Remark 4.6. For a $2 n \times 2 n$ skew symmetric matrix $X^{s k}$, we have $W \subset \operatorname{Ann}\left(\operatorname{Pf}\left(X^{s k}\right)\right)$.

Lemma 4.7. For the generic skew symmetric $2 n \times 2 n$ matrix $X^{\text {sk }}$, we have

$$
W=\operatorname{Ann}\left(P_{4}\left(X^{s k}\right)\right) \cap S_{2}^{s k}
$$

Proof. The monomials of type (a) and (b) correspond to unacceptable monomials discussed earlier and are linearly independent from any binomial in (c). The binomials in (c) are linearly independent by Theorem 4.3. Hence, we have

$$
\begin{aligned}
\operatorname{dim}_{\mathrm{k}}(W) & =2\left(\begin{array}{c}
2 n \\
4
\end{array}\right)+\left(2 n^{2}-n\right)(2 n-2)+2 n^{2}-n \\
& =\left(\begin{array}{c}
2 n^{2}-n+1 \\
2
\end{array}\right)-\left(\begin{array}{c}
2 n \\
4
\end{array}\right) .
\end{aligned}
$$

According to Remark 1.4, we have

$$
\operatorname{dim}_{\mathrm{k}}\left(\operatorname{Ann}\left(P_{4}\left(X^{s k}\right)\right)\right) \cap S_{2}^{s k}=\operatorname{dim}_{\mathrm{k}} S_{2}^{s k}-\operatorname{dim}_{\mathrm{k}}\left(P_{4}\left(X^{s k}\right)\right) .
$$

So we have

$$
\operatorname{dim}_{\mathrm{k}}\left(\operatorname{Ann}\left(P_{4}\left(X^{s k}\right)\right)\right) \cap S_{2}^{s k}=\left(\begin{array}{c}
2 n^{2}-n+1 \\
2
\end{array}\right)-\left(\begin{array}{c}
2 n \\
4
\end{array}\right) .
$$

Using equations (4.3) and (4.4), we obtain

$$
\operatorname{dim}_{\mathrm{k}}(W)=\operatorname{dim}_{\mathrm{k}}\left(\operatorname{Ann}\left(P_{4}\left(X^{s k}\right)\right)\right) \cap S_{2}^{s k} .
$$

On the other hand, evidently we have

$$
\left.W \subset \operatorname{Ann}\left(P_{4}\left(X^{s k}\right)\right)\right) \cap S_{2}^{s k} .
$$

Using equations (4.5) and (4.6), we have

$$
W=\operatorname{Ann}\left(P_{4}\left(X^{s k}\right)\right) \cap S_{2}^{s k} .
$$


Lemma 4.8. Let $X^{\text {sk }}$ be a $2 n \times 2 n$ skew symmetric matrix $(n \geq 2)$. We have

$$
S_{n-2} \circ \operatorname{Pf}\left(X^{s k}\right)=P_{4}\left(X^{s k}\right) \subset R_{2}^{s k} .
$$

Proof. First we show

$$
S_{n-2} \circ \operatorname{Pf}\left(X^{s k}\right) \supset P_{4}\left(X^{s k}\right) .
$$

We use induction on the size of the matrix.

The first step is $2 n=6$. We denote by $f=\left[i_{1}, i_{2}, i_{3}, i_{4}\right] \in P_{4}\left(X^{s k}\right)$ the Pfaffian of the sub matrix with the rows and columns $i_{1}, i_{2}, i_{3}$ and $i_{4}$. We have $\left(\begin{array}{l}6 \\ 4\end{array}\right)=15$ choices for $f$. For any of these choices we get the Pfaffian of a $2 \times 2$ sub matrix of the form

$$
\left(\begin{array}{cc}
0 & x \\
-x & 0
\end{array}\right)
$$

as the coefficient of $f$ in the Pfaffian of the matrix $X^{s k}$. So, if we differentiate the $6 \times 6$ Pfaffian with respect to that variable $x$, we get the $4 \times 4$ Pfaffian $f=\left[i_{1}, i_{2}, i_{3}, i_{4}\right]$.

Assume that equation (4.7) holds for the generic skew symmetric $(2 n-2) \times(2 n-2)$ matrix. We want to show it holds for the $2 n \times 2 n$ generic skew symmetric matrix. The Pfaffian of the skew symmetric $2 n \times 2 n$ matrix $X^{s k}$ can be computed recursively as

$$
P f\left(X^{s k}\right)=\sum_{i=2}^{i=2 n}(-1)^{i} x_{1 i}^{s k} \operatorname{Pf}\left(X_{\hat{1} \hat{i}}^{s k}\right),
$$

where $X_{\hat{1} \hat{i}}^{s k}$ denotes the matrix $X^{s k}$ with both the first and the $i$ th rows and columns removed. So $X_{\hat{1} \hat{i}}^{s k}$ is a $(2 n-2) \times(2 n-2)$ matrix and equation (4.7) holds for it. So, for each choice of $\left[i_{1}, i_{2}, i_{3}, i_{4}\right]$ of the matrix $X_{\hat{1} \hat{i}}^{s k}$ we can find $n-3$ variables of $X_{\hat{1} \hat{i}}^{s k}$ such that differentiating $\operatorname{Pf}\left(X_{\hat{1} \hat{i}}^{s k}\right)$ with respect to those variables gives us the Pfaffian $\left[i_{1}, i_{2}, i_{3}, i_{4}\right]$. If we call those variables $a_{1}, \ldots, a_{n-3}$ and add $x_{1 i}^{s k}$ to our set of $n-3$ variables, we will have a set of $n-2$ variables. Then, using equation (4.8), if we differentiate $\operatorname{Pf}\left(X^{s k}\right)$ with respect to those $n-2$ variables, we will get the Pfaffian $\left[i_{1}, i_{2}, i_{3}, i_{4}\right]$. Since we could write the recursive formula for the Pfaffian with respect to any other row or column, the result follows. 
For the opposite inclusion to equation (4.7), we have

$$
W \subset\left(\operatorname{Ann}\left(P f\left(X^{s k}\right)\right)\right)_{2} \subset\left(\operatorname{Ann}\left(P_{4}\left(X^{s k}\right)\right)\right)_{2} .
$$

But we have shown in Lemma 4.7 that

$$
W=\left(\operatorname{Ann}\left(P_{4}\left(X^{s k}\right)\right)_{2} .\right.
$$

So we have

$$
\left(\operatorname{Ann}\left(P f\left(X^{s k}\right)\right)\right)_{2}=\left(\operatorname{Ann}\left(P_{4}\left(X^{s k}\right)\right)\right)_{2} .
$$

By Remark 1.4, we have

$$
\left(\operatorname{Ann}\left(P f\left(X^{s k}\right)\right)\right)_{2}=\operatorname{Ann}\left(S_{n-2} \circ\left(P f\left(X^{s k}\right)\right)\right) .
$$

Hence, we have

$$
S_{n-2} \circ \operatorname{Pf}\left(X^{s k}\right)=P_{4}\left(X^{s k}\right) .
$$

Recall that we denote by $P_{2 k}\left(X^{s k}\right)$ the vector subspace of $R^{s k}$ spanned by the $2 k$-Pfaffian minors of $X^{s k}$ (Definition 4.2 ).

Lemma 4.9. For $1 \leq k \leq n-1$, we have

$$
S_{k} \circ\left(P f\left(X^{s k}\right)\right)=P_{2 n-2 k}\left(X^{s k}\right) .
$$

Proof. First, we want to show

$$
S_{k} \circ\left(P f\left(X^{s k}\right)\right) \subset P_{2 n-2 k}\left(X^{s k}\right) .
$$

We use induction on $k$. For $k=1$, we need to prove

$$
S_{1} \circ\left(\operatorname{Pf}\left(X^{s k}\right)\right) \subset P_{2 n-2}\left(X^{s k}\right),
$$

so we need to show that, for any monomial $y_{i j} \in S_{1}$, we have

$$
y_{i j} \circ\left(P f\left(X^{s k}\right)\right) \subset P_{2 n-2}\left(X^{s k}\right) .
$$

It is enough to show that the above inclusion holds for $y_{12}$. Using 
equation (4.8), we have

$$
\begin{aligned}
y_{12} \circ\left(\operatorname{Pf}\left(X^{s k}\right)\right) & =y_{12} \circ \sum_{i=2}^{i=2 n}(-1)^{i} x_{1 i}^{s k} \operatorname{Pf}\left(X_{\hat{1} \hat{i}}^{s k}\right) \\
& =\operatorname{Pf}\left(X_{\hat{1} \hat{2}}^{s k}\right)+\sum_{i=3}^{i=2 n}(-1)^{i} x_{1 i}^{s k} \operatorname{Pf}\left(X_{\hat{1} \hat{i}}^{s k}\right) \in P_{2 n-2}\left(X^{s k}\right) .
\end{aligned}
$$

So, indeed,

$$
S_{1} \circ\left(P f\left(X^{s k}\right)\right) \subset P_{2 n-2}\left(X^{s k}\right) .
$$

Next assume $S_{k} \circ\left(P f\left(X^{s k}\right)\right) \subset P_{2 n-2 k}\left(X^{s k}\right)$. We want to show

$$
S_{k+1} \circ\left(P f\left(X^{s k}\right)\right) \subset P_{2 n-2 k-2}\left(X^{s k}\right) .
$$

We have

$$
\begin{aligned}
S_{k+1} \circ\left(P f\left(X^{s k}\right)\right) & =S_{1} \circ\left(S_{k} \circ\left(P f\left(X^{s k}\right)\right)\right. \\
& \subset S_{1} \circ\left(P_{2 n-2 k}\left(X^{s k}\right)\right. \\
& \subset P_{2 n-2 k-2}\left(X^{s k}\right) .
\end{aligned}
$$

For the other inclusion, we again use induction on $k$. First we show that the inclusion holds for $k=1$. Let $\eta \in P_{2 n-2}\left(X^{s k}\right)$ be a $(2 n-2) \times(2 n-2)$ Pfaffian minor of $X^{s k}$. Corresponding to $\eta$, there exists a $2 \times 2$ matrix of the form

$$
\left(\begin{array}{cc}
0 & x \\
-x & 0
\end{array}\right)
$$

where $x$ is not in the $2 n-2$ rows and columns of $\eta$. If we differentiate the Pfaffian of $X^{s k}$ with respect to $x$, we will get $\eta$. So we have $\eta \in S_{1} \circ\left(\operatorname{Pf}\left(X^{s k}\right)\right)$.

Next assume $P_{2 n-2 k}\left(X^{s k}\right) \subset S_{k} \circ\left(\operatorname{Pf}\left(X^{s k}\right)\right)$. We have

$$
\begin{aligned}
P_{2 n-2 k-2}\left(X^{s k}\right) & \subset S_{1} \circ\left(P_{2 n-2 k}\left(X^{s k}\right)\right) \\
& \subset S_{1} \circ\left(S_{k} \circ\left(\operatorname{Pf}\left(X^{s k}\right)\right)\right) \\
& =S_{k+1} \circ\left(\operatorname{Pf}\left(X^{s k}\right)\right) .
\end{aligned}
$$

Thus, by induction, the equality holds. 
Recall that $(W)$ is the ideal of $S^{s k}$ spanned by degree 2 elements of type (a), (b) and (c) as in Definition 4.5.

Proposition 4.10. For the $2 n \times 2 n$ generic skew symmetric matrix $X^{s k}$, we have

$$
(W)_{n}=\operatorname{Ann}\left(\operatorname{Pf}\left(X^{s k}\right)\right) \cap S_{n}^{s k}
$$

Proof. Let $2 \leq k \leq n$. By Remark 1.4 and Lemma 4.9, we have

(1) $W \circ P f\left(X^{s k}\right)=0 \Leftrightarrow W \circ S_{n-2}^{s k} P f\left(X^{s k}\right)=0 \Leftrightarrow W \circ P_{4}\left(X^{s k}\right)=0$.

(2) $\left(\operatorname{Ann}\left(P f\left(X^{s k}\right)\right)\right) \cap S_{2}=W \Rightarrow S_{k-2} W \circ\left(S_{n-k} \circ \operatorname{Pf}\left(X^{s k}\right)\right)=0$.

$$
\begin{aligned}
& \Longrightarrow S_{k-2}(W) \circ P_{2 k}\left(X^{s k}\right)=0 . \\
& \Longrightarrow(W)_{k} \circ P_{2 k}\left(X^{s k}\right)=0 .
\end{aligned}
$$

Therefore, for all integers $k, 2 \leq k \leq n$, we have

$$
(W)_{k} \subset \operatorname{Ann}\left(P_{2 k}\left(X^{s k}\right)\right) \cap S_{k}^{s k} .
$$

We need to show

$$
(W)_{n} \supset \operatorname{Ann}\left(P f\left(X^{s k}\right)\right) \cap S_{n}^{s k} .
$$

We use induction on $n$. For $n=1,2$, we have the $2 \times 2$ and $4 \times 4$ skew symmetric matrices, and the equality is easy to see.

Now we want to show that the proposition holds for $n=3$. We use Remark 2.8. Let $\eta$ be a binomial in $\operatorname{Ann}\left(\operatorname{Pf}\left(X^{s k}\right)\right) \cap S_{3}^{s k}$. Without loss of generality, we can write

$$
\eta=y_{12} y_{34} y_{56}-y_{\sigma(1) \sigma(2)} y_{\sigma(3) \sigma(4)} y_{\sigma(5) \sigma(6)},
$$

where $\sigma \in S_{6}, \operatorname{sgn}(\sigma)=1$, and we have $\sigma(1)<\sigma(3)<\sigma(5)$, $\sigma(1)<\sigma(2), \sigma(3)<\sigma(4)$ and $\sigma(5)<\sigma(6)$.

If the two terms of the binomial $\eta$ have a common factor, then, without loss of generality, we can assume that the common factor is $y_{12}$. So we can write $\eta$ as

$$
\eta=y_{12}\left(y_{34} y_{56}-y_{\sigma(3) \sigma(4)} y_{\sigma(5) \sigma(6)}\right) \text {. }
$$

But, by the definition of $(W)_{3}$, the monomial $y_{34} y_{56}-y_{\sigma(3) \sigma(4)} y_{\sigma(5) \sigma(6)}$ is included in $W$ since it is of the form (c). So we have $\eta \in(W)_{3}$.

On the other hand, assume that the two terms of $\eta$, i.e., $y_{12} y_{34} y_{56}$ and $y_{\sigma(1) \sigma(2)} y_{\sigma(3) \sigma(4)} y_{\sigma(5) \sigma(6)}$ do not have any common 
factor. We can add and subtract another term of the Pfaffian $\tau=y_{\beta(1) \beta(2)} y_{\beta(3) \beta(4)} y_{\beta(5) \beta(6)}$ such that $\beta$ is a permutation in $S_{6}$, and we have $\beta(1)<\beta(3)<\beta(5), \beta(1)<\beta(2), \beta(3)<\beta(4)$ and $\beta(5)<\beta(6)$. Also, $\tau$ has one common factor with $y_{12} y_{34} y_{56}$ and one common factor with $y_{\sigma(1) \sigma(2)} y_{\sigma(3) \sigma(4)} y_{\sigma(5) \sigma(6)}$. Without loss of generality, we can take $\beta(5)=5, \beta(6)=6$ and $\beta(1)=\sigma(1), \beta(2)=$ $\sigma(2)$. So we have

$$
\eta-\tau+\tau=\eta-y_{\sigma(1) \sigma(2)} y_{\beta(3) \beta(4)} y_{5,6}+y_{\sigma(1) \sigma(2)} y_{\beta(3) \beta(4)} y_{5,6} \cdot
$$

Hence, we have

$$
\begin{aligned}
\eta= & y_{5,6}\left(y_{12} y_{34}-y_{\sigma(1) \sigma(2)} y_{\beta(3) \beta(4)}\right) \\
& +y_{\sigma(1) \sigma(2)}\left(y_{\beta(3) \beta(4)} y_{5,6}-y_{\sigma(3) \sigma(4)} y_{\sigma(5) \sigma(6)}\right) .
\end{aligned}
$$

But, by the definition of $W$, we know that $y_{12} y_{34}-y_{\sigma(1) \sigma(2)} y_{\beta(3) \beta(4)}$ and $y_{\beta(3) \beta(4)} y_{5,6}-y_{\sigma(3) \sigma(4)} y_{\sigma(5) \sigma(6)}$ are both elements of $W$ of type (c). So we have $\eta \in(W)_{3}$.

When $n$ is larger than 3 , then by the induction assumption, we can assume that the proposition holds for all integers $k \leq n-1$. Again, we use Remark 2.8. Assume $b=b_{1}+b_{2}$ is of degree $n$. If the two terms, $b_{1}$ and $b_{2}$, are monomials in $S^{s k}$ and have a common factor $l$, i.e., $b_{1}=l a_{1}$ and $b_{2}=l a_{2}$, then $b=l\left(a_{1}+a_{2}\right)$ where $a_{1}$ and $a_{2}$ are of degree at most $n-1$. By the induction assumption, the proposition holds for the binomial $a_{1}+a_{2}$, i.e., $a_{1}+a_{2} \in W_{n-1}$; hence, we have

$$
b=l\left(a_{1}+a_{2}\right) \in l(W)_{n-1} \subset(W)_{n} .
$$

If the two terms, $b_{1}$ and $b_{2}$, do not have any common factor, then with the same method as above, we can rewrite the binomial $b$ by adding and subtracting a term $m$ of degree $n$, which has a common factor $m_{1}$ with $b_{1}$ and a common factor $m_{2}$ with $b_{2}$. Then we will have

$$
b_{1}+b_{2}=b_{1}+m+b_{2}-m=m_{1}\left(c_{1}+m^{\prime}\right)+m_{2}\left(c_{2}-m^{\prime \prime}\right),
$$

where $b_{1}=m_{1} c_{1}, m=m_{1} m^{\prime}=m_{2} m^{\prime \prime}$ and $b_{2}=m_{2} c_{2}$. Since $c_{1}+m^{\prime}$ and $c_{2}-m^{\prime \prime}$ are of degree at most $n-1$, the induction assumption yields

$$
b_{1}+b_{2}=m_{1}\left(c_{1}+m^{\prime}\right)+m_{2}\left(c_{2}-m^{\prime \prime}\right) \in(W)_{n} .
$$


This completes the induction step and the proof of the proposition.

Corollary 4.11. For $1 \leq k \leq n$, we have

$$
(W)_{k}=\operatorname{Ann}\left(P f\left(X^{s k}\right)\right) \cap S_{k}^{s k} .
$$

We also have $(W)_{n+1}=S_{n+1}^{s k}$.

Proof. Using equation (4.11), we only need to show that

$$
\operatorname{Ann}\left(P f\left(X^{s k}\right)\right) \cap S_{k}^{s k} \subset(W)_{k} .
$$

By Remark 1.4 and Lemma 4.9, we have

$$
\left(\operatorname{Ann}\left(\operatorname{Pf}\left(X^{s k}\right)\right)\right)_{k}=\left(\operatorname{Ann}\left(S_{n-k} \circ \operatorname{Pf}\left(X^{s k}\right)\right)\right)_{k}=\left(\operatorname{Ann}\left(P_{2 k}\left(X^{s k}\right)\right)\right)_{k} .
$$

Now if we label the $2 k \times 2 k$ Pfaffians of $X^{s k}$ by $f_{1}, \ldots, f_{s}$ we have

$$
\operatorname{Ann}\left(P_{2 k}\left(X^{s k}\right)\right)_{k}=\left(\operatorname{Ann}\left\langle f_{1}, \ldots, f_{s}\right\rangle\right)_{k}=\left(\bigcap_{i=1}^{i=s}\left(\operatorname{Ann}\left(f_{i}\right)\right)\right)_{k} .
$$

Let $R^{i}$ denote the ring in the variables of $f_{i}$ and $W(i)$ the vector space analogue of $W$ in those $R$ variables corresponding to $f_{i}$. By Proposition 4.10, we have

$$
(W(i))_{k}=\operatorname{Ann}\left(f_{i}\right) \cap S_{k}^{i} .
$$

So we have

$$
\operatorname{Ann}\left(P f\left(X^{s k}\right)\right) \cap S_{k}^{s k} \subset(W)_{k}
$$

To prove the second part, it is easy to see that every monomial of degree larger than $n$ will be unacceptable, of type (a) or (b), as in $W$. Then we have $(W)_{n+1}=S_{n+1}^{s k}$.

Theorem 4.12. Let $X^{s k}$ be a generic skew symmetric $2 n \times 2 n$ matrix. Then the apolar ideal Ann $\left(P f\left(X^{s k}\right)\right)$ is the ideal $(W)$ that is generated in degree 2 by the vector space $W$ of Definition 4.5.

Proof. This follows directly from Proposition 4.10 and Corollary 4.11. 
Corollary 4.13. Let $X^{s k}$ be a $2 n \times 2 n$ generic skew symmetric matrix. We have

$$
2^{2 n-2} \leq \operatorname{cr}\left(\operatorname{Pf}\left(X^{s k}\right)\right) \leq 2^{2 n-1}
$$

Proof. By the Ranestad-Schreyer proposition, Corollary 4.4 and Theorem 4.12, we have

$$
\begin{aligned}
\operatorname{cr}\left(\operatorname{Pf}\left(X^{s k}\right)\right) & \geq \frac{1}{2} \operatorname{dim}\left(S^{s k} / \operatorname{Ann}\left(\operatorname{Pf}\left(X^{s k}\right)\right)\right) \\
& =\frac{1}{2}\left(2^{2 n-1}\right)=2^{2 n-2} .
\end{aligned}
$$

The second inequality is true by equation (3.2).

Remark 4.14. For $n \geq 5$, it can be easily seen that the lower bound for the cactus rank given by Corollary 4.12 is larger than $l_{\text {diff }}=\left(\begin{array}{c}2 n \\ 2 t_{0}\end{array}\right)$, where $t_{0}=\lfloor n / 2\rfloor$.

Theorem 4.15. Let $X^{s}$ be a generic symmetric $2 n \times 2 n$ matrix. Then the apolar ideal Ann $\left(H f\left(X^{s}\right)\right)$ is generated in degree 2, and the inequality (4.13) also holds for $\left(H f\left(X^{s}\right)\right)$.

Proof. By the definition of the hafnian, it is easy to see that none of the diagonal elements appear in $H f\left(X^{s}\right)$, so for $1 \leq i \leq 2 n$ we have

$$
y_{i i} \circ H f\left(X^{s}\right)=0 .
$$

Hence, without loss of generality, we can restrict our discussion to the case where $X^{s}$ is a generic zero-diagonal symmetric matrix. By changing the Pfaffians to hafnians, and vice versa, the proof follows directly from the proofs that we have for the Pfaffian of a generic skew symmetric matrix.

5. Gröbner bases. In Section 2 we have shown that, for $A$ a generic $n \times n$ matrix Ann $(\operatorname{det}(A))=\left(\mathcal{P}_{D}+\mathcal{U}_{D}\right)$. In [16], Laubenbacher and Swanson give a Gröbner basis for the ideal of $2 \times 2$ permanents of a matrix. In this section, we first review their result (Theorem 5.2), then state our result for the ideal Ann $(\operatorname{det}(A))$ and prove it independently (Theorem 5.3). 
Definition 5.1. ([16, page 197]). Let $D=\left(d_{i j}\right)$ be the matrix of the differential operators as defined in Section 1. A monomial order on the $d_{i j}$ is diagonal if, for any square submatrix of $D$, the leading term of the permanent (or of the determinant) of that submatrix is the product of the entries on the main diagonal. An example of such an order is the lexicographic order defined by:

$$
d_{i j}<d_{k l} \text { if and only if } l>j \text { or } l=j \text { and } k>i .
$$

Throughout this section, we use a lexicographic diagonal ordering.

Theorem 5.2. ([16, page 197]). The following collection $G$ of polynomials is a minimal reduced Gröbner basis for $\mathcal{P}_{D}$, with respect to any diagonal ordering:

(1) The subpermanents $d_{i j} d_{k l}+d_{k j} d_{i l}, i<k, j<l$;

(2) $d_{i_{1} j_{1}} d_{i_{1} j_{2}} d_{i_{2} j_{3}}, i_{1}>i_{2}, j_{1}<j_{2}<j_{3}$;

(3) $d_{i_{1} j_{1}} d_{i_{2} j_{2}} d_{i_{2} j_{3}}, i_{1}>i_{2}, j_{1}<j_{2}<j_{3}$;

(4) $d_{i_{1} j_{1}} d_{i_{2} j_{1}} d_{i_{3} j_{2}}, i_{1}<i_{2}<i_{3}, j_{1}>j_{2}$;

(5) $d_{i_{1} j_{1}} d_{i_{2} j_{2}} d_{i_{3} j_{2}}, i_{1}<i_{2}<i_{3}, j_{1}>j_{2}$;

(6) $d_{i_{1} j_{1}}^{e_{1}} d_{i_{2} j_{2}}^{e_{2}} d_{i_{3} j_{3}}^{e_{3}}, i_{1}<i_{2}<i_{3}, j_{2}>j_{3}, e_{1} e_{2} e_{3}=2$.

Monomials of type (2)-(6) in the above theorem are in the ideal generated by all unacceptable monomials.

Theorem 5.3. The collection of unacceptable degree 2 monomials and $2 \times 2$ subpermanents of $D$, form a Gröbner basis for $\operatorname{Ann}(\operatorname{det}(A))$ with respect to any diagonal ordering. In particular, the algebra $\mathfrak{A}_{A}=$ $S /(\operatorname{Ann}(\operatorname{det}(A))$ is Koszul (see [8, page 2]).

Proof. We will denote $\mathcal{U}_{D}$ and $\mathcal{P}_{D}$ by $\mathcal{U}$ and $\mathcal{P}$, respectively in the following, where $D$ is understood.

The elements of $(\mathcal{U}+\mathcal{P})$ generate $\operatorname{Ann}(\operatorname{det}(A))$. Since $\mathcal{U}$ is a set of monomials, it is already Gröbner. We use Buchberger's algorithm to find a Gröbner basis for $\mathcal{P}+\mathcal{U}$. We consider several cases: 
(a) Let $F$ and $G$ be distinct permanents of $D$. Let $F=a_{i k} a_{j l}+a_{i l} a_{j k}$ and $G=a_{u z} a_{v w}+a_{u w} a_{v z}$ be two permanents in $\mathcal{P}$.

$$
F=\operatorname{perm}\left(\begin{array}{cc}
a_{i k} & a_{i l} \\
a_{j k} & a_{j l}
\end{array}\right) .
$$

and

$$
G=\operatorname{perm}\left(\begin{array}{ll}
a_{u z} & a_{u w} \\
a_{v z} & a_{v w}
\end{array}\right) .
$$

Let $f_{1}=a_{i k} a_{j l}$ be the leading term of $F$, and let $g_{1}=a_{u z} a_{v w}$ be the leading term of $G$ with respect to the given diagonal ordering. Denote the least common multiple of $f_{1}$ and $g_{1}$ by $h_{11}$. Let

$$
S(F, G)=\left(h_{11} / f_{1}\right) F-\left(h_{11} / g_{1}\right) G=a_{u z} a_{v w} a_{i l} a_{j k}-a_{i k} a_{j l} a_{u w} a_{v z} .
$$

Now, using the multivariate division algorithm, reduce all the $S(F, G)$ relative to the set of all permanents. When there is no common factor in the initial terms of $F$ and $G$ the reduction is zero, one can use $F$ and $G$ again as we show. First, we reduce $S(F, G)$ dividing by $F \in \mathcal{P}$, so we will have

$$
S(F, G)+a_{u w} a_{v z}\left(a_{i k} a_{j l}+a_{i l} a_{j k}\right)=a_{u z} a_{v w} a_{i l} a_{j k}+a_{u w} a_{v z} a_{i l} a_{j k} .
$$

Then we reduce the result using $G$ this time, so we will have

$$
a_{u z} a_{v w} a_{i l} a_{j k}+a_{u w} a_{v z} a_{i l} a_{j k}-a_{i l} a_{j k}\left(a_{u z} a_{v w}+a_{u w} a_{v z}\right)=0 .
$$

Therefore, we have shown that, for all pairs $F, G$ of distinct permanents of $D$, the $S$-polynomials $S(F, G)$ reduce to zero with respect to $\mathcal{P}$.

(b) Let $F=a_{i k} a_{j l}+a_{i l} a_{j k}$ and $G=a_{i k} a_{j m}+a_{i m} a_{j k}$ be two permanents so that their initial terms have a common factor. We have

$$
S(F, G)=a_{i l} a_{j k} a_{j m}-a_{i m} a_{j k} a_{j l} \in \mathcal{U}
$$

(c) Let $F=a_{i m} a_{j n}+a_{i n} a_{j m}$ be a permanent and $M=a_{t k} a_{t l}$ an unacceptable monomial. We have

$$
S(F, M)=a_{t k} a_{t l} a_{j m} a_{i n} \in \mathcal{U} .
$$

(d) Let $F=a_{i l} a_{j m}+a_{i m} a_{j l}$ be a permanent and $M=\left(a_{k n}\right)^{2}$ an unacceptable monomial. We have

$$
S(F, M)=a_{i m} a_{j l}\left(a_{k n}\right)^{2} \in \mathcal{U} .
$$


(e) Let $F=a_{i l} a_{j m}+a_{i m} a_{j l}$ be a permanent and $M=\left(a_{i l}\right)^{2}$ an unacceptable monomial which has a common factor with the initial term of $F$. We have

$$
S(F, M)=a_{i l} a_{i m} a_{j l} \in \mathcal{U} .
$$

(f) Let $F=a_{i l} a_{j m}+a_{i m} a_{j l}$ be a permanent and $M=a_{j n} a_{k n} \mathrm{n}$ unacceptable monomial. We have

$$
S(F, M)=a_{i m} a_{j l} a_{j n} a_{k n} \in \mathcal{U} .
$$

This exhausts all possibilities, so the generating set $\mathcal{P}+\mathcal{U}$ is itself a Gröbner basis by Buchberger's algorithm.

Corollary 5.4. The collection of unacceptable degree 2 monomials and the $2 \times 2$ minors of $D$, form a Gröbner basis for Ann $(\operatorname{perm}(A))$ with respect to any diagonal ordering. In particular, the algebra $\mathfrak{B}_{A}=$ $S /(\operatorname{Ann}(\operatorname{perm}(A))$ is Koszul (see [8, page 2]).

Proof. The proof is similar to that given for Theorem 5.3.

\subsection{Discussion of the connected sum.}

Definition 5.5. ([18]). A polynomial $F$ in $r$ variables is a connected sum if we can write $F=F^{\prime}+F^{\prime \prime}$ with $F^{\prime}$ and $F^{\prime \prime}$ in $r^{\prime}$ and $r^{\prime \prime}$ variables, where $r^{\prime}+r^{\prime \prime}=r$.

Let $A$ be a generic $2 \times 2$ matrix. We can write the determinant $A$ is a sum of two polynomials in complementary sets of variables.

Proposition 5.6 (Buczyńska, et al. [5]). If a form $F$ of degree $d$ is a connected sum, then the apolar ideal has a minimal generator in degree $d$. (The converse does not hold.)

In particular, since the generic determinant and permanent of size $n \geq 3$ have their annihilating ideals generated in degree 2, therefore they are not connected sums. This is also true for the Pfaffian of skew symmetric matrices and hafnian of symmetric matrices of size $n \geq 6$. 
Acknowledgments. This paper is from a Ph.D. dissertation at Northeastern University. I am deeply grateful to my advisor Prof. Anthony Iarrobino, whose help, stimulating ideas and encouragement helped me in working on this problem and writing this paper. I am very thankful to Prof. Zach Teitler for suggesting this problem and his helpful comments, and also to Prof. Aldo Conca and Prof. Larry Smith for their valuable comments and suggestions.

\section{REFERENCES}

1. J. Alexander and A. Hirschowitz, Polynomial interpolation in several variables, J. Alg. Geom. 4 (1995), 201-222.

2. A. Bernardi, P. Marques and K. Ranestad, Computing the cactus rank of a general form, arXiv:1211.7306 (2012).

3. A. Bernardi and K. Ranestad, On the cactus rank of cubic forms, J. Symb. Comp. 50 (2013), 291-297.

4. W. Bruns and A. Conca, Gröbner bases and determinantal ideals, in Commutative algebra, singularities and computer algebra, J. Herzog et al., eds., NATO Sci. Math. Phys. Chem. 115 (2003), 9-66.

5. W. Buczyńska, J. Buczyński, J. Kleppe and Z. Teitler, Apolarity and direct sum decomposability of polynomial, arXiv: 1307.3314 (2013).

6. W. Buczyńska, J. Buczyński and Z. Teitler, Waring decompositions of monomials, J. Alg. 378 (2013), 45-57.

7. E. Carlini, M.V. Catalisano and A.V. Geramita, The solution to Waring problem for monomials, J. Alg. 370 (2012), 5-14.

8. A. Conca, Koszul algebras and Gröbner bases of quadrics, arXiv: 0903.2397v1 (2001).

9. A.V. Geramita, Inverse systems of fat points: Waring's problem, secant varieties of veronese varieties and parameter spaces for Gorenstein ideals, Queen's Papers Pure Appl. Math. 102 (1996), 3-104.

10. D.M. Goldschmidt, Algebraic functions and projective curves, Grad. Texts Math. 215, Springer-Verlag, New York, 2003.

11. D.R. Grayson and M.E. Stillman, Macaulay2, A software system for research in algebraic geometry, available at http://www.math.uiuc.edu/Macaulay2/.

12. J. Herzog and N. Trung, Gröbner bases and multiplicity of Determinantal and Pfaffian ideals, Adv. Math. 96 (1992), 1-37.

13. A. Iarrobino and V. Kanev, Power sums, Gorenstein algebras, and determinantal varieties, Springer Lect. Not. Math. 1721 (1999), 345+xxvii pages.

14. M. Ishikawa, H. Kawamuko and S. Okada, A Pfaffian-hafnian analogue of Borchardt's identity, Electr. J. Comb. 12 (2005).

15. J.M. Landsberg and Z. Teitler, On the ranks and border ranks of symmetric tensors, Found. Comp. Math. 10 (2010), 339-366. 
16. R.C. Laubenbacher and I. Swanson, Permanental ideals, J. Symb. Comp. 30 (2000), 195-205.

17. F.S. Macaulay, The algebraic theory of modular systems, Cambridge Mathematical Library, Cambridge University Press, Cambridge, 1916; (reprinted London, 1994).

18. D. Meyer and L. Smith, Poincare duality algebras, Macaulay's dual systems, and Steenrod operations, Cambr. Tracts Math. 167, Cambridge University Press, Cambridge, 2005.

19. K. Ranestad and F.-O. Schreyer, On the rank of a symmetric form, J. Alg. 346 (2011), 340-342.

20. M. Shafiei, Apolarity for determinants and permanents of generic symmetric matrices, arXiv:1303.1860 (2013).

21. R. Stanley, Enumerative combinatorics, Volume 1. Second edition. Cambr. Stud. Adv. Math. 49, Cambridge University Press, Cambridge, 2012.

22. Z. Teitler, Maximum Waring ranks of monomials, arXiv:1309.7834v1 (2013).

Department of Mathematics, Northeastern University, Boston, MA 02115 Email address: sepideh.shafiee@gmail.com 